Philosophische Fakultät, Universität Ljubljana

luka.horjak@ff.uni-lj.si

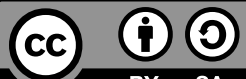

BY SA

\title{
ALTHOCHDEUTSCHE GRAMMATIK. I. LAUT- UND FORMLEHRE. 16. AUFLAGE
}

Wilhelm Braune, Frank Heidermanns (2018). Althochdeutsche Grammatik. I. Laut- und Formlehre. 16. Auflage. Neubearbeitet von Frank Heidermanns. Berlin/Boston: De Gruyter. ISBN 978-3-11-051510-7, 557 Seiten, 29,95€.

\section{$0 \quad$ EINLEITUNG}

Vor uns liegt das langlebige Referenzwerk der deutschen historischen Grammatik, das bereits seine 16. Auflage erlebte. Die Althochdeutsche Grammatik: I. Laut- und Formlehre umfasst Phonologie und Morphologie der germanischen Dialekte zwischen dem 7. Jh. und der 2. Hälfte des 11. Jahrhunderts im hochdeutschen Sprachraum. Die hohe Anzahl der Auflagen spricht für die kontinuierliche Aktivität auf dem Forschungsgebiet und für die unübertreffliche Qualität des Werkes. Die Erstauflage wurde im Jahr 1886 von dem wichtigen Junggrammatiker und Mediävisten Wilhelm Braune herausgegeben. Sie ist Teil der Sammlung kurzer Grammatiken germanischer Dialekte und bildet mit anderen historischen Grammatiken, z. B. die Mittelhochdeutsche Grammatik von Herman Paul ( $\left.{ }^{1} 1881,{ }^{25} 2007\right)$, die Althochdeutsche Grammatik: II. Syntax von Richard Schrodt (2004), die Gotische Grammatik von Wilhelm Braune $\left({ }^{1} 1880,{ }^{20} 2004\right)$ u. a., die Basisbibliothek vor allem für diachrone LinguistInnen und MediävistInnen. Diese Rezension beruht auf dem Vergleich mit der von Ingo Reiffenstein bearbeiteten 15. Auflage (2004).

Das Werk ist seit der Erstauflage paragrafenweise aufgebaut. Im Laufe der Zeit wurden existierende Paragrafen aufgebessert, neuere Inhalte wurden als Zwischenparagrafen, die mit einem zusätzlichen Buchstaben gekennzeichnet sind, eingelegt. In der Neuauflage wurde die Gestalt des Werkes noch benutzerfreundlicher gestaltet. Jetzt steht vor jeder Paragrafnummer noch der Buchstabe des Hauptkapitels (E für Einleitung, L für Lautlehre, F für Formlehre) und die Nummer des Unterkapitels, z. B. L 2. Vokalismus (§ 9-77a), L 2.1.6.1. Ablaut (§ 50), L 3. Konsonantismus (§ 78-191), L 3.1.6. Grammatischer Wechsel (§ 100-102) usw. 
Im Bereich von Laut- und Formlehre sind mehrere Quellenverweise als zuvor zu finden, da das Werk sowohl als Lehrbuch als auch Referenzwerk der althochdeutschen Sprachstufe dient. Die Quellen der Neuauflage wurden mit der Zeitzer Beichte, dem Fragment des Computus und den Sankt Galler Fragmenten der Vetus Latina erweitert. Das Quellenverzeichnis befindet sich jetzt am Anfang des Werkes. In der Neuauflage sind linguistische Kennzeichnungen für Grapheme $(<>)$, Phoneme (//) und Allophone/ Laute ([]) konsequent gebraucht. Weitere Anweisungen auf Sekundärliteratur befinden sich nicht mehr unter den Anmerkungen einzelner Paragrafen, sondern im eigenen Absatz unter dem Paragrafen.

In der Einleitung ( 1 1a, Anm. 3.) findet man eine Aufzählung der wichtigen digitalen Ressourcen (Handschriftenportale, Wörterbücher, Quellen und Texte) zum Althochdeutschen, neu sind auch Vergleiche des Althochdeutschen mit dem Altsächsischen (§ 2a). Besonders hilfreich ist die neue Liste der wichtigen phonologischen und morphologischen Charakteristika einzelnen althochdeutschen Dialekten - des Oberdeutschen im Allgemeinen, Alemannischen, Bairischen und Fränkischen (§ 6a).

Die Übersicht der Vokalgrapheme wurde mit folgenden Diagraphen erweitert: $<$ aa $>,<$ ao $>$, $<\mathrm{au}>,<\mathrm{ee}>,<\mathrm{ii}>,<\mathrm{iy}>,<\mathrm{oe}>,<_{\mathrm{oo}}>,<_{\mathrm{oy}}>$ und $<\mathrm{uu}>$ (vgl. §13-21). Neben der graphischen Bearbeitung der Tabellen, die übersichtlicher gestaltet sind, wurden auch neue tabellarische Darstellungen hinzugefügt, wie z. B. die Quantitätskorrelationen bzw. Vokalminimalpaare (§ 24). Die Anmerkungen zum primären Umlaut (§ 27) wurden auf das aktuelle Forschungsstand gebracht, hinzugefügt wurden Umlaut von /a/ über/e/ bis zu/i/ (Anm. 7.) und Umlaut von /ai/ zu /ē/ (Anm. 8.). Die Phonemsymbole wurden in der Neuauflage überarbeitet, so steht /e/ für das kurze germanische $e$ (früher /ë/), das Lautsymbol für das lange germanische $/ \overline{\mathrm{e}}\left(\overline{\mathrm{e}}^{-1}\right) /$ wurde mit $/ \overline{\mathrm{x}}\left(\overline{\mathrm{e}}_{1}\right) /$ ersetzt, parallel dazu auch das lange germanische $/ \overline{\mathrm{e}}\left(\overline{\mathrm{e}}^{2}\right) /$ mit $/ \overline{\mathrm{e}}\left(\overline{\mathrm{e}}_{2}\right) /$, um die qualitative Unterschiede zwischen den beiden germanischen $e$-Lauten zu bewahren. Bei dem a-Umlaut (§ 52) wurde der Terminus Senkung neben der Brechung benutzt. Terminologisch wurde das Kapitel Die Vokale der nicht starktonigen Silben zu Die Vokale der Nebensilben (§ 54) und Die Vokale der Vorsilben zu Die Vokale der Präfixe (§ 70) umbenannt; mit dem Oberbegriff für die Endsilben, Mittelsilben und Präfixe, die keine Hauptbetonung tragen können, wurde die Kontinuität der Terminologie bewahrt. Bei den Sprossvokalen (§ 69) wurde neben der eingedeutschten Vokalentfaltung der Terminus das Anaptyxe statt früher Svarabhakti verwendet. 
Der Kapitel des Konsonantismus wurde durch die Übersicht der ostfränkischen Konsonantenphonemen ( $\$ 80)$ erweitert. Nach der tabellarischen Darstellung folgen die konsonantischen Quantitätsoppositionen zwischen den einfachen und geminierten bzw. langen Konsonanten. Besonders wichtig sind neue Anmerkungen zur (vermutlichen) Aussprache des Konsonanten, z. B. des $r$-Lautes im Althochdeutschen (Anm. 4. des $\S 80$ ). Die phonologische Transkription des Werkes nähert sich mit seiner Überarbeitung dem Internationalen phonetischen Alphabet. Das Symbol des stimmhaften velaren Frikativen, der durch Spirantisierung aus dem stimmlosen $/ \chi /$ im In- und Auslaut schon im Urgerm. entstanden ist, wurde zu $/ \mathrm{g} /$, früher $/ \gamma /$ - dadurch ist zwar die Unterscheidung zwischen der Entwicklung des ide. $/ \mathrm{k} /$ und des ide. $/ \mathrm{g}^{\mathrm{h}} /$ verschwunden, wurde aber das Inventars der Konsonantenphonemen im phonetischen Sinne vereinheitlicht (vgl. § 81). Neu sind die Paragrafen zum germanischen $/ \mathrm{k}^{\mathrm{w}} /$ (§ 146a) und zum germanischen $/ \mathrm{h}^{\mathrm{w}} /(\S 154 \mathrm{a})$. Der Übersicht der Konsonantengrapheme wurde mit weiteren Sequenzen der Grapheme erweitert: $<\mathrm{fh}>,<\mathrm{gch}>,<\mathrm{gu}>,<\mathrm{hk}>,<\mathrm{hqu}>,<\mathrm{hs}>,<\mathrm{phf}>,<\mathrm{sh}>,<\mathrm{ssc}>(\S 173, \S 176-178$, $\S 184, \S 187)$, neu ist der Paragraf 190a zum Graphem $<\mathrm{y}>$.

Der Terminus Doppelkonsonanten wurde mit langen Konsonanten (Geminaten) ( $\S$ 82) bzw. geminierte Konsonanten (§91) ersetzt, es geht nämlich um längere und nicht doppelte Aussprache des Konsonanten. Wegen Vereinheitlichung wurde der veraltete Terminus Sonorlaute mit Sonanten ersetzt (§ 104). Besonders lobenswert ist die Arbeit, die an der Klarheit des Textes geleistet wurde. An vielen Stellen wurden Beschreibungen der Reihenfolgen der Phoneme präzisiert und systematisiert, z. B. bei der Anm. 3. des $\S$ 118 steht in der Neuausgabe „Zur Folge $/ \mathrm{r} /+/ \mathrm{j} / “$, in der 15. Ausgabe stand „Das j nach r“. Der Terminus Gutturale wurde mit dem Terminus Velare ersetzt ( $\$ 140)$, da es im Althochdeutschen keine Palatale gab (das $j$ galt im Ahd. als Halbvokal [i]); der Terminus Guttural fungiert als Oberbegriff für velare und palatale Laute. Einige Paragrafen wurden stark erweitert, z. B. zum germanischen /1/ (§ 122).

FORMLEHRE

Im Oberkapitel Formlehre wurden einzelne Unterkapiteln systematisch umbenannt, z. B. statt ja-Stämme steht Stämme auf -ja-oder statt Ehemalige germ. -iz-/-az-Stämme steht Flexion mit germ. *iz-/-az u. a. Im Bereich der Terminologie wird Wurzelvokal statt früher Stammvokal verwendet, aber inkonsequent (vgl. § 245). Die Paragrafe 204 und 205 wurden mit der Kategorie der Nomina, die sowohl als Maskulina als auch Neutra vorkommen, z. B. hrēo, rēo 'Leichnam', klēo 'Klee', sou 'Saft', tou 'Tau', trëso 'Schatz', erweitert. Dem Paragrafen 204 wurden auch weitere Beispiele, z. B. brīo ,'Brei', dëo 'Diener', $b \bar{u}$ 'Bau, Wohnung' usw., hinzugefügt. Die Beschreibung der Deklination der 
Stämme auf -wō- wurde erweitert, in der früheren Ausgabe gab es nur ein Hinweis auf die Deklination der reinen ō-Stämmen (§ 212). Bei der i-Deklination des Femininums wurde das Paradigma des Wortes anst 'Gunst' mit dem Paradigma kraft 'Kraft' ersetzt (§ 218). Die Deklination der Maskulina auf $-n-(\S 222)$ wurde durch die Kategorie der aus schwach flektierten Adjektiven substantivierten Personenbezeichnungen, z. B. mennisko 'Mensch' und wīzago 'Prophet', und den Lehnwortschatz, z. B. pfaffo 'Pfaffe', ergänzt. Neu ist die Auseinandersetzung mit dem ide. Wortbildungstypen Nomina ohne Suffix bzw. Wurzelnomina (§ 237a).

Bei der Deklination der Adjektiva auf $-a-/ \overline{-}-$ und bei der schwachen Deklination der Adjektiva wurde der Beispiel blint bzw. blinto 'blind' mit dem Beispiel jung bzw. jungo 'jung' ersetzt ( $\$ 248, \S 255)$, bei der Steigerung der Adjektiva wurde das Paradigma für die Komparation mit dem Beispiel jungiro 'jünger' (früher rëhtiro 'richtiger') ajouriert (§ 262).

Der Paragraf 267 zu Adjektivadverbien wurde mit weiteren Beispielen und Anmerkungen ergänzt. Der ehemalige Paragraf 270 wurde entzweit, sodass der neue Paragraf 269a jetzt als eine Einleitung zu den Zahlwörtern fungiert. Das Paradigma der Possessivpronomina hat in der Neuausgabe eine tabellarische Illustration bekommen (§ 285).

Bei der Konjugation des ahd. Verbes wurde der Modus, der noch in der 15. Ausgabe Optativ hieß, in den Konjunktiv umbenannt (vgl. § 301), um die terminologische Kontinuität zum Mittel- und Neuhochdeutschen zu sichern und dem 2. Band der Althochdeutschen Grammatik: II. Syntax (Schrodt 2004) zu entsprechen. Die Benennungen der beiden Tempora wurden in der Neuausgabe eingedeutscht, Präsens statt Praesens und Präteritum statt Praeteritum. Zur Illustration der Konjugation verschiedener Verbkassen wurde eine Tabelle mit dem fränkischen Lautstand des 9. Jh. hinzugefügt (vgl. § 328a).

\section{SCHLUSSBEMERKUNGEN}

Die 16. Ausgabe der Althochdeutschen Grammatik: I. Laut- und Formlehre bietet allen, die sich für die deutsche Sprachgeschichte interessieren, das Grundwissen zum Thema und zugleich einen Wegweiser mit umfangreichen und aktualisierten Quellen- und Sekundärliteraturverzeichnis. Besonders lobenswert sind zahlreiche Stellen, die auf die Uneinigkeit der SprachwissenschaftlerInnen aufweisen, das Paradebeispiel wäre die Datierung der 2. (hochdeutschen) Lautverschiebung. Das Werk soll nach der Vollendung des Leipziger Althochdeutschen Wörterbuches neugestaltet werden und damit nur noch als Grammatik fungieren. 